\title{
CO2.3
}

\section{ANDAMENTO STAGIONALE ATIPICO DELLA MALATTIA DI LYME IN SUD ITALIA}

\author{
Vallone G., Noto A., Vallone A ., Apuzzo G., \\ Greco F., Tenuta R., Guaglianone L., Giraldi C. \\ U.O. Malattie Infettive, Microbiologia e Virologia \\ Ospedale Annunziata AO Cosenza
}

\section{Introduzione:}

La malattia di Lyme è una zoonosi molto comune nei paesi dell'emisfero settentrionale che ha un tipico andamento stagionale essendo più frequente in estate, anche se in ogni periodo dell'anno è possibile registrare dei casi.

\section{Obiettivi:}

Valutare l'andamento stagionale della malattia di Lyme in Calabria.

\section{Metodi:}

Studio prospettico condotto dal marzo 2000 al marzo 2005 su soggetti con malattia di Lyme, rispondente alla definizione di caso dei CDC, consecutivamente valutati, che non avevano soggiornato al di fuori della Calabria nei 3 mesi precedenti la diagnosi. Per ogni soggetto sono stati riportati: un numero personale progressivo, il sesso, l'età, la data della diagnosi, la data di inizio dei sintomi, le caratteristiche cliniche. Sono stati eseguiti test sierologici (Lyme EIA IgG-IgM e Western blot Marlblot, distribuiti dalla Arnika - Milano) su campioni di sangue prelevati al momento della prima visita, e dopo 1 e 3 mesi.

\section{Risultati:}

Sono stati osservati 41 soggetti con malattia di Lyme (3 nel primo anno dello studio, 9 nel secondo, 15 nel terzo, 8 nel quarto e 6 nel quinto) con le caratteristiche necessarie per rientrare nella definizione di caso dei CDC. Di questi, 9 sono stati osservati nel trimestre gennaio-marzo $(21,9 \%), 11(26,8 \%)$ nel trimestre aprile-giugno, nessun caso nel trimestre luglio-settembre e $21(51,2 \%)$ nel trimestre ottobre-dicembre. Dei 41 casi, $38(91,6 \%)$ presentavano eritema migrante, un caso artrite, uno cardite e uno neuroborreliosi. Il tasso complessivo di positività all' EIA è stato del $17.3 \% \mathrm{e}$ del $13 \%$ con conferma alla Western blot. Il tasso complessivo di positività alla Western blot è stato del $60.8 \%$.

\section{Conclusioni:}

Dai risultati del nostro studio emerge che la distribuzione stagionale della malattia di Lyme in Calabria segue un andamento diverso rispetto a quanto si verifica nelle regioni del nord, come suggerito dalla netta concentrazione dei casi nel trimestre ottobre-dicembre e dall'assenza di casi nel trimestre luglio-settembre. 